\title{
Ibuprofen-Maltodextrin Interaction: Study of Enantiomeric Recognition and Complex Characterization
}

\section{Claudia Garnero, Carolina Aloisio, Marcela Longhi}

Departamento de Farmacia, Facultad de Ciencias Químicas, Universidad Nacional de Córdoba, Ciudad Universitaria, Córdoba, Argentina.

Email: garneroc@fcq.unc.edu.ar, caloisio@fcq.unc.edu.ar,mrlcor@fcq.unc.edu.ar

Received September $30^{\text {th }}, 2012$; revised November $9^{\text {th }}, 2012$; accepted December $19^{\text {th }}, 2012$

\begin{abstract}
The interaction between ibuprofen and maltodextrins with different dextrose equivalent was studied in solution and solid state in order to investigate the effect on the solubility of ibuprofen and to determine their usefulness in terms of chiral recognition. Apparent binding constants were calculated using nuclear magnetic resonance spectroscopy experiments and solubility studies. The results showed an increase in the apparent solubility of ibuprofen in the presence of maltodextrins that depended on their ionization state. The freeze-drying method was used to prepare solid complexes, while physical mixtures were obtained by simple blending. These solid systems were characterized in the solid state using differential scanning calorimetry, thermogravimetric analysis, Fourier Transform-Infrared spectroscopy, scanning electron microscopy and X-ray diffractometry. Detailed nuclear magnetic resonance studies provided evidence of the influence of the type and concentration of the maltodextrin host on the chiral recognition of racemic ibuprofen, indicating that these linear ligands act as chiral selectors.
\end{abstract}

Keywords: Ibuprofen; Maltodextrin; Chiral Recognition; Complex Characterization; Nuclear Magnetic Resonance Spectroscopy; Solubility Studies

\section{Introduction}

To be therapeutically active, drugs must be able to dissolve in body fluids and permeate membranes in order to travel to other body compartments. Ibuprofen (IBP), 4isobutyl-2-phenyl-propionic acid, is a well-known nonsteroidal anti-inflammatory drug (NSAID) widely used for the treatment of pain, inflammation, arthritis, fever and dysmenorrhea. However, its bioavailability is relatively low after oral administration, since it presents poor solubility in water [1]. In the literature data related to the biopharmaceutics classification system (BCS) IBP was assessed to be a class II drug. Therefore an increase in its solubility is paramount to improve the dissolution rate from solid dosage forms and thereby its therapeutic activity. IBP, as in the case of others profens (ketoprofen, naproxen and flurbiprofen) of the 2-aryl propionic acid family of nonsteroidal anti-inflammatory drugs, displays an asymmetric carbon atom on the propionic acid side chain, leading to the existence of the enantiomeric pairs: $\mathrm{S}(+)$-IBP and $\mathrm{R}(-)$-IBP (see structures in Figure 1). However, despite IBP being marketed as a racemate, only the $\mathrm{S}(+)$-IBP enantiomer is implicated in the inhibition of prostaglandin synthesis and anti-inflammatory events [2]. In the present study, the solubility of the model drug IBP was improved and the chiral discrimination by the formation of complexes, was investigated.

In previous works, cyclodextrins (CDs) have been employed to promote the dissolution of the drugs through their solubilisation and also to differentiate chiral drug enantiomers, which may be included inside the CD cavity. In particular, $\beta$-CD has been studied as a chiral host in the discrimination of IBP isomers by means of atomistic molecular mechanics simulations and 2D NMR experiments [3], as well as for the determination of IBP enantiomers in human plasma and urine by spectrofluorimetry [4]. In addition, $\alpha-\mathrm{CD}, \beta-\mathrm{CD}$ and $\gamma-\mathrm{CD}$ were used to determine the enantiomeric composition of samples of IBP by chemometric analysis of UV spectral data [5].

In addition, maltodextrins (MDs) have been used as chiral selectors $[6,7]$. They are obtained by acid and/or enzymatic controlled hydrolysis of starch, but to a lower extent than that required to produce starch syrups. It is clear, then that MD are complex mixtures of high and low molecular weight materials, which contain linear amylose and branched amylopectin degradation products, therefore they are considered as D-glucose polymers joined by $\alpha-(1,4)$ and $\alpha-(1,6)$ linkages. The extent of 
<smiles>CC(C)Cc1ccc(C(C)C(=O)O)cc1</smiles>

$\Gamma$

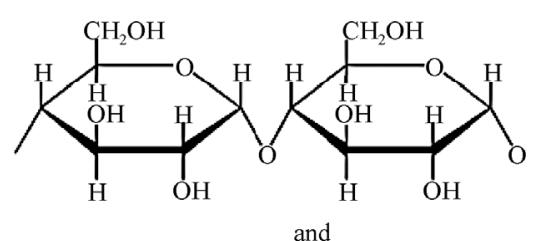

]$_{n}$
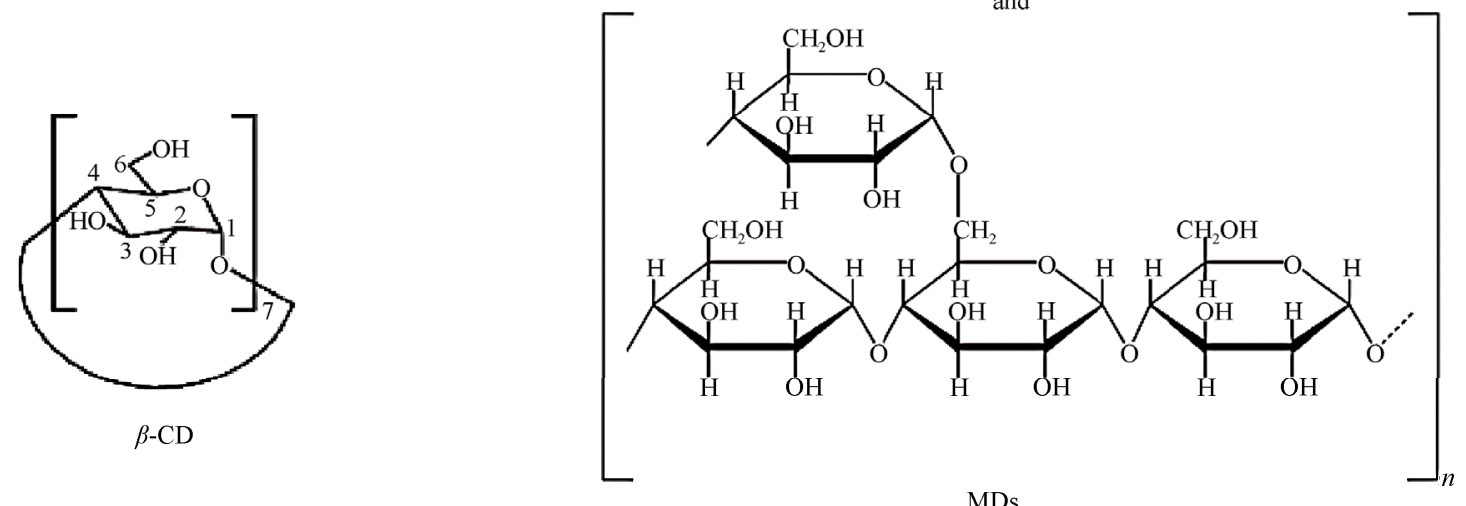

Figure 1. Chemical structure of S(+)-IBP, R(-)-IBP, MDs and $\beta$-CD.

starch degradation in a MD is indicated by the Dextrose Equivalent value (DE) that evaluates the content of reducing-end groups, and is inverse value of average degree of polymerisation (DP) of anhydro glucose units. The nature of the starch hydrolysis, and the process used will have an important influence on the composition and properties of final product, that have different physicochemical properties like solubility, freezing temperature, viscosity, etc. depending on the DE value $[8,9]$. Their typical structure is shown in Figure 1.

The MDs are endowed with the capability of complexation to various classes of compounds, with the hostguest one being the most common interaction for starch derivatives. The conformational change from a flexible coil to a helix form in the presence of a guest, which depends on the size of the complexing molecules, is believed to be essential for interactions to occur. The helical structure is hydrophobic inside, as in the CD cavity, but MDs have considerably more flexible entities than CDs, which is an important property in chiral recognition [10].

NMR based methods, such as ${ }^{1} \mathrm{H}-\mathrm{NMR}$, have been used to evaluate enantiomeric recognition and to determine the enantiomeric excess of chiral compounds. The development of new types of chiral selectors with higher efficiencies is of primary importance in the development of a versatile and accurate method for the resolution of enantiomeric forms in chiral drugs. In particular, the NMR techniques could provide considerable information about theoretical models of the enantioseparation of enantiomeric IBP with cyclic and linear oligosaccharides, which may be applied for the chiral recognition process in capillary electrophoresis and chromatographic separa- tion.

The aim of this study was to evaluate the effect of adding MDs with different DE value to enhance the solubility of IBP and to determine their usefulness in terms of IBP chiral recognition. Detailed NMR studies of the chiral recognition of racemic IBP comparing cyclic oligosaccharide $(\beta-\mathrm{CD})$ and linear polysaccharides (MDs) are reported. The parameters believed to affect the enantioresolution of IBP, such as type and concentration of the chiral selector, were investigated. Additionally, nuclear magnetic resonance ( ${ }^{1} \mathrm{H}-\mathrm{NMR}$ and 2D-ROESY), differential scanning calorimetry (DSC), thermogravimetric analysis (TGA), Fourier Transform-Infrared spectroscopy (FT-IR), scanning electron microscopy (SEM) and X-ray diffractometry (XRD) were utilized to analyze the physicochemical properties of the complexes obtained.

\section{Materials and Methods}

\subsection{Materials}

Racemic Ibuprofen was provided by Parafarm (Argentina), S-Ibuprofen was obtained from Sigma-Aldrich ${ }^{\circledR}$ (USA), and $\beta$-CD and Glucidex ${ }^{\circledR}$ IT 19 (DE19) maltodextrin were gifts from Ferromet agent of Roquette (Lestrem, France). Maltodextrin (DE17) was provided by Todo Droga (Argentina). $\mathrm{D}_{2} \mathrm{O}$ (deuterium content 99.9\%) was obtained from Aldrich ${ }^{\circledR}$ (USA) and $\mathrm{NaOD}(40 \%$ solution in $\mathrm{D}_{2} \mathrm{O}$, deuterium content $99 \%$ ) was provided by SIGMA ${ }^{\circledR}$ (St Louis, MO, USA). All other chemicals were of analytical grade. A Millipore Milli Q Water Purification System (Millipore, Bedford, MA, USA) generated the water used in these studies. 


\subsection{Phase Solubility Studies}

The solubility measurements were performed according to the method of Higuchi and Connors [11]. An excess of IBP was added to buffered aqueous solutions ( $\mathrm{pH} 2.0$, 4.6 and 7.0) containing increasing concentrations of MDs with DE 17 (MD17, 0.5 - $7.7 \mathrm{mM}$ ) and DE 19 (MD19, $0.5-8.8 \mathrm{mM}$ ). IBP, in the absence of MDs, was used to determine the intrinsic solubility. The suspensions were sonicated for $15 \mathrm{~min}$ (ULTRASONIC LC $30 \mathrm{H} \mathrm{Elma)}$ and then placed for $72 \mathrm{~h}$ in a $25.0^{\circ} \mathrm{C} \pm 0.1^{\circ} \mathrm{C}$ constant temperature water bath [Haake DC10 thermostat (Haake, Paramus, NJ, USA)]. These suspensions were then sonicated at several time intervals. After the equilibrium was reached, the solid IBP remaining was removed by filtration through a $0.45 \mu \mathrm{m}$ membrane filter (Millipore, USA). The clear solutions were then suitably diluted and analyzed by UV spectrophotometry (SHIMADZU UV-160A spectrophotometer, VA Howe, UK) at $222 \mathrm{~nm}$.

The studies were performed using McIlvaine buffers prepared from $0.2 \mathrm{M} \mathrm{Na}_{2} \mathrm{HPO}_{4}$ and $0.1 \mathrm{M}$ citric acid, with an ionic strength of 0.5 .

The apparent $1: 1$ stability constants $\left(\mathrm{K}_{\mathrm{c}}\right)$ were calculated from the straight line portion of the phase solubility diagrams, according to the following equation [11]:

$$
\mathrm{K}_{\mathrm{c}}=\text { slope } / \mathrm{S}_{0}(1-\text { slope })
$$

where slope is the value found in the linear regression and $\mathrm{S}_{0}$ is the solubility of IBP determined in the absence of MDs at each $\mathrm{pH}$ value.

\subsection{Nuclear Magnetic Resonance (NMR)}

Due to its poor solubility in $\mathrm{D}_{2} \mathrm{O}$ at low $\mathrm{pD}$ values not permitting NMR studies, all IBP solutions were prepared in $\mathrm{D}_{2} \mathrm{O}$ with an adjustment to $\mathrm{pD} 11$ using appropriate amounts of $\mathrm{NaOD}$.

All experiments were performed on a Bruker ${ }^{\circledR}$ Avance II High Resolution Spectrometer equipped with a Broad Band Inverse probe (BBI) and a Variable Temperature Unit (VTU), at $298 \mathrm{~K}$ using $5 \mathrm{~mm}$ sample tubes. The NMR data were processed with the Bruker TOPSPIN 2.0 software and ${ }^{1} \mathrm{H}$-NMR spectra were obtained at 400.16 MHz. The chemical shifts $(\delta)$ were reported as ppm, and the residual solvent signal $(4.80 \mathrm{ppm})$ was used as the internal reference. Induced changes in the ${ }^{1} \mathrm{H}-\mathrm{NMR}$ chemical shifts $(\Delta \delta)$ for IBP caused by its interaction with MD were calculated according to the following equation:

$$
\Delta \delta=\delta_{\text {complex }}-\delta_{\text {free }}
$$

Assuming that the IBP-MD complexes had a 1:1 stoichiometry, the $\mathrm{K}_{\mathrm{c}}$ values were calculated based on Scott's modification of the Benesi-Hildebrand equation $[12,13]$. The slope of the plot [ligand $] / \Delta \delta_{\text {obs }}$ against [ligand] is equal to $1 / \Delta \delta_{\mathrm{c}}$ and the intercept with the vertical axis is $1 / \mathrm{K}_{\mathrm{c}} \Delta \delta_{\mathrm{c}}$, thereby allowing $\mathrm{K}_{\mathrm{c}}$ to be estimated.

2D-ROESY: the interactions were studied by two-dimensional Rotating frame Overhauser experiments (2DROESY), with spinlock for mixing phase sensitive, using $180 \times 180$-x pulses for polarization transfer. The spectra were measured with a relaxation delay of $2 \mathrm{~s}, \mathrm{p} 15$ pulse for ROESY spinlock of $20 \mathrm{~ms}$ and 14, spinlock loop, $(\mathrm{p} 15 / \mathrm{p} 25 \times 2)-400$. Before Fourier transformation, the matrix was zero filled to 4096 (F2) by 2048 (F1) and Gaussian apodization functions were applied in both dimensions. Samples had a 1:1 mixture of IBP and MDs (MD17 and MD19, respectively) in $\mathrm{D}_{2} \mathrm{O}$, and the concentration of each component was $15 \mathrm{mM}$.

\subsection{Preparation of the Complexes}

The preparation of the solid IBP-MD complexes with a 1:1 molar ratio was performed by the freeze-drying method. Appropriate amounts of each component were suspended in water and sonicated at $25.0^{\circ} \mathrm{C} \pm 0.1^{\circ} \mathrm{C}$ constant water temperature until the drug was completely dissolved. Solutions were frozen at $-40^{\circ} \mathrm{C}$ for $24 \mathrm{~h}$ to ensure complete solidification before freeze-drying was started (Freeze Dye 4.5 Labconco corp., Kansas City, MI). Physical mixtures were prepared by mixing the corresponding components uniformly in a mortar at a 1:1 molar ratio.

\subsection{Differential Scanning Calorimetry (DSC) and Thermogravimetric Analysis (TGA)}

The DSC curves of the samples were obtained with a DSC TA 2920, and the TGA curves were recorded on a TG TA 2920. The samples were placed in aluminium hermetic pans, and the experiments were carried out under nitrogen gas flow at a heating rate of $10^{\circ} \mathrm{C} / \mathrm{min}$ and temperature range of $25^{\circ} \mathrm{C}-350^{\circ} \mathrm{C}$. Data were obtained and processed using the TA Instruments Universal Analysis 2000 software.

\subsection{Fourier Transform-Infrared Spectroscopy (FT-IR)}

The FT-IR spectra were recorded on a Nicolet 5 SXC FT-IR Spectrophotometer (Madison, WI, USA), and potassium bromide disks were obtained by compressing the powder.

\subsection{Scanning Electron Microscopy (SEM)}

The shape and surface characteristics of the raw materials, the binary complexes and the physical mixtures were investigated and photographed using a scanning electron microscope LEO Model EVO 40XVP. The samples were fixed on a brass stub using double-sided aluminium tape. 
To improve the conductivity, samples were gold-coated under vacuum employing a sputter coater PELCO Model 3. The magnification selected was sufficient to be able to appreciate in detail the general morphology of the samples under study.

\subsection{X-Ray Diffractometry (XRD)}

X-ray powder diffraction patterns were recorded by a Rigaku Miniflex 2000 X-ray diffractometer, with Ni filtered $\mathrm{Cu}-\mathrm{K} \alpha$ radiation and a scanning rate from $5^{\circ}$ to $40^{\circ}$ $(2 \theta)$ at steps of $0.05^{\circ}$, a voltage of $30 \mathrm{~kW}$ and a current of $15 \mathrm{~mA}$ for the generator. Data were obtained using Standard Measurement software.

\subsection{Statistical Analyses}

Treatment of the data were performed on Origin Pro 8, involved the use of ANOVA. Continuous variables were expressed as median \pm median absolute deviation.

\section{Results and Discussion}

\subsection{Phase Solubility Studies}

The effect of MD17 and MD19 on the solubility of IBP was investigated at $\mathrm{pH}$ values where the drug molecule was either unionized $(\mathrm{pH} 2.0)$ or ionized $(\mathrm{pH} \mathrm{7.0)}$, and also for a $\mathrm{pH}$ near $\mathrm{pK}_{\mathrm{a}}(\mathrm{pH}$ 4.6). The solubility values
(Table 1) showed that MD17 at $\mathrm{pH} 2.0$ produces a significant increase in the solubility of IBP, compared with those in buffer solution without MD. In contrast, we observed a lower effectiveness on the interaction between IBP and the MDs at $\mathrm{pH} 4.6$ and 7.0, and between IBP and MD19 at $\mathrm{pH}$ 2.0. Our results at $\mathrm{pH} 2.0$ showed relevant differences deriving from the DE value of the MDs, which demonstrated different IBP-MD interactions for both MDs. This was related to the extent of starch degradation in the MD, which indicated that IBP-MD binding only occurred when the chain length of the MD molecules exceeded a particular value. In addition, the phase solubility diagrams, obtained by plotting the changes in guest solubility as a function of MD concentrations, showed different profiles depending on the MD and $\mathrm{pH}$ used.

The interaction of the IBP-MD17 system (Figure 2(a)) in buffer solution at $\mathrm{pH} 2.0$ displayed a typical $\mathrm{A}_{\mathrm{L}}$ type solubility curve [11], indicating the formation of a soluble complex of presumably a 1:1 stoichiometry. On other hand, the diagrams obtained at $\mathrm{pH}$ values of 4.6 and 7.0 did not correspond to either type A or type B classes. These plots showed an immediate and progressive decrease in the apparent concentration of IBP in solution for increasing MD concentration.

The phase solubility profiles of the IBP-MD19 system are presented in Figure 2(b), with the solubility diagram

Table 1. Summary of phase solubility studies.

\begin{tabular}{|c|c|c|c|c|c|c|c|}
\hline \multirow{2}{*}{$\mathrm{pH}$} & \multirow{2}{*}{$\mathrm{S}_{0}(\mathrm{mg} / \mathrm{mL})$} & \multicolumn{3}{|c|}{ MD17 } & \multicolumn{3}{|c|}{ MD19 } \\
\hline & & $\mathrm{S}_{\max }(\mathrm{mg} / \mathrm{mL})$ & $\mathrm{K}_{\mathrm{c}}\left(\mathrm{M}^{-1}\right)$ & $\mathrm{CE}$ & $\mathrm{S}_{\max }(\mathrm{mg} / \mathrm{mL})$ & $\mathrm{K}_{\mathrm{c}}\left(\mathrm{M}^{-1}\right)$ & $\mathrm{CE}$ \\
\hline 2.0 & 0.03 & 0.16 & $420 \pm 4$ & 0.08 & 0.04 & $61.7 \pm 0.7$ & 0.008 \\
\hline 4.6 & 0.09 & 0.06 & - & - & 0.1 & $13 \pm 1$ & 0.006 \\
\hline 7.0 & 2.3 & 1.9 & - & - & 2.71 & - & - \\
\hline
\end{tabular}

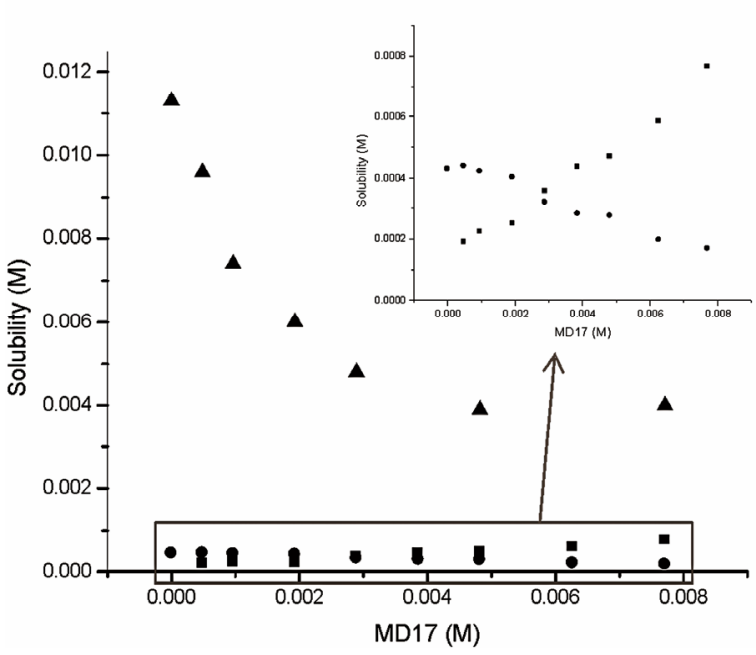

(a)

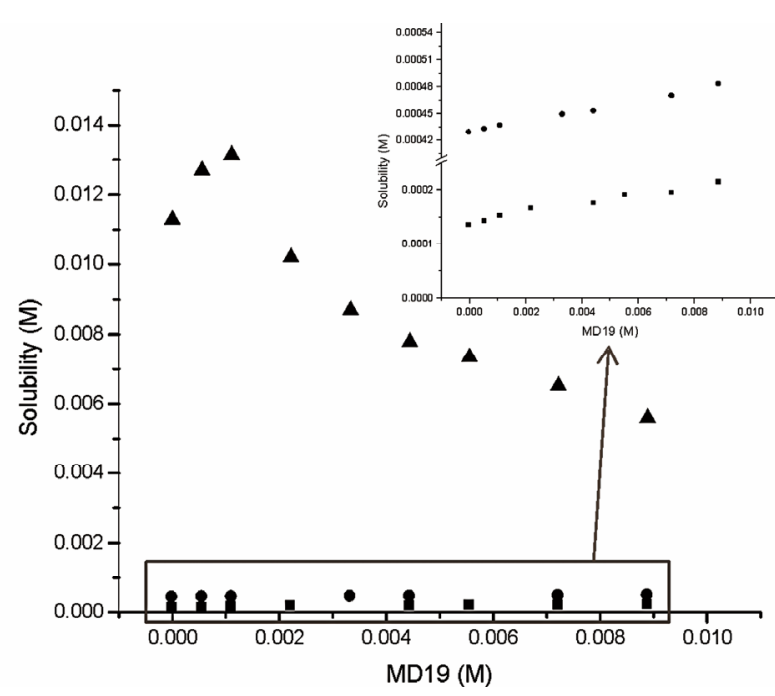

(b)

Figure 2. Effect of (a) MD17 and (b) MD19 on the solubility of IBP at $25.0^{\circ} \mathrm{C}$ in buffer pH 2.0 (匹), buffer pH $4.6(\bullet)$ and buffer pH 7.0 ( $\Delta)$ solutions. 
obtained in buffer solution at $\mathrm{pH} 2.0$ being classified as an $A_{N}$ type. This curve may be originated from both an alteration in the effective nature of the solvent in the presence of large concentrations of ligand and a selfassociation of MD19 at higher concentrations. On the other hand, the solubility of IBP increased linearly with a rise in the concentration of MD19 at $\mathrm{pH} 4.6$, which is characteristic of an $A_{L}$ type diagram. Considering this curve, it can be seen that the apparent solubility of IBP increased due to the formation of a soluble complex, whereas the diagram constructed at $\mathrm{pH} 7.0$ corresponded to a $\mathrm{B}_{\mathrm{s}}$ type curve. The complex exhibited a higher solubility than the guest molecule, but its limit was reached within the tested MD concentration range. The increase in the amount of available MD molecules did not lead to a rise in solubility, indicating that all guest molecules had been converted into a less soluble complex [14].

The calculated $\mathrm{K}_{\mathrm{c}}$ values for IBP-MD17 and IBPMD19 systems at $\mathrm{pH} 2.0$ were $420 \pm 4 \mathrm{M}^{-1}$ and $61.7 \pm 0.7$ $\mathrm{M}^{-1}$, respectively. These values clearly demonstrate, within experimental error, that IBP in buffer solution at $\mathrm{pH} 2.0$ had the highest affinity for MD17. Also, the interaction of IBP with MD19 at $\mathrm{pH} 4.6$ was lower $\left(\mathrm{K}_{\mathrm{c}}=13 \pm 1 \mathrm{M}^{-1}\right)$ than at $\mathrm{pH} 2.0$, showing that neutral IBP $(\mathrm{pKa}=4.55,[1])$ exhibited a higher affinity for the host than the ionic species had, which indicates a significant contribution of the hydrophobic effect towards complex stability.

The results showed that the increase in the apparent solubility of IBP in the presence of MDs depended on the ionization state. At pH 2.0, MD17 increased drug solubility more than MD19, whereas at $\mathrm{pH} 7.0$ the opposite occurred.

According to these results and taking into account a previous work using CDs [15], it can be affirmed that the complexation of the drug with MD is better when the unionized form of the drug is present, while the total solubility achieved is significantly increased when the drug is fully ionized.

\subsection{Nuclear Magnetic Resonance (NMR) of Selector-Solute Interactions}

The interaction of IBP with $\beta$-CD, MD17 and MD19 was first evaluated by analyzing the differences in the chemical shifts $(\delta)$ of their hydrogen atoms with respect to the corresponding uncomplexed signals. The full assignment of the IBP protons and the changes observed after the addition of each selector at a 1:1 ratio are summarized in Table 2. The signal of IBP C proton was not included in the data evaluation due to resonance overlap caused by MD signals.

Upon the addition of chiral selectors, the ${ }^{1} \mathrm{H}-\mathrm{NMR}$ spectra of racemic IBP showed pronounced changes typical of complexation in all IBP protons, with either shielding (with $\beta$-CD and MD19) or deshielding (with MD17) effects. Furthermore, by comparing the shifts of the ${ }^{1} \mathrm{H}-$ NMR spectra of S-IBP with those of the complexes, a splitting of the signals was observed in the complexes spectra as a result of the different binding modes of the IBP enantiomers (Figure 3).

Table 2. Complexation induced chemical shifts $(\Delta \delta)$ observed for racemic IBP in the presence of $\beta$-CD, MD17 and MD19 at a 1:1 molar ratio.

\begin{tabular}{ccccc}
\hline \multicolumn{2}{c}{ IBP } & \multicolumn{3}{c}{$\Delta \delta$} \\
\hline Proton & $\delta_{\text {free }}$ & $\beta$-CD & MD17 & MD19 \\
\hline A & 7.2674 & -0.0665 & 0.0294 & -0.0453 \\
B & 7.2117 & -0.1933 & -0.0197 & -0.0835 \\
D & 2.4686 & -0.0162 & 0.0157 & -0.0388 \\
E & 1.8364 & -0.0148 & 0.0341 & - \\
F & 1.3861 & -0.0261 & 0.0378 & -0.0377 \\
G & 0.8736 & 0.0333 & 0.0553 & -0.0251 \\
\hline
\end{tabular}

$\Delta \delta=\delta_{\text {complex }}-\delta_{\text {free }}$.

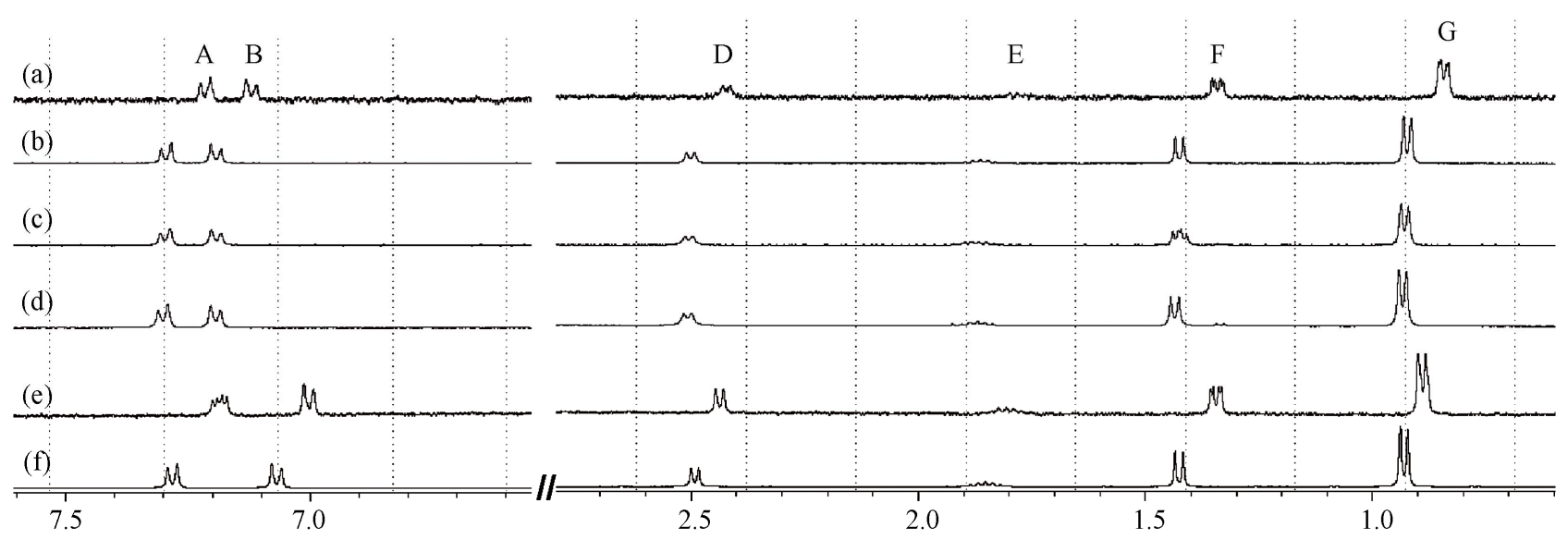

Figure 3. ${ }^{1} \mathrm{H}-\mathrm{NMR}$ spectra expansions of: (a) IBP-MD19; (b) S(+)-IBP-MD19; (c) IBP-MD17; (d) S(+)-IBP-MD17; (e) IBP- $\beta$-CD and (f) $S(+)-I B P-\beta-C D$ at a 1:1 molar ratio. 
These results reveal evidence of interactions of IBP with each selector, which suggests the existence of different modes of interaction between the enantiomeric forms of IBP and both MDs.

\subsubsection{Effect of the Type and Concentration of Chiral Selectors on Enantiomer Discrimination}

In the present study, the chiral recognition of IBP by cyclic and linear oligosaccharides, such as $\beta$-CD, MD17 and MD19, was tested by NMR. Two types of shifts for racemic drug resonance signals could be observed after the addition of a chiral selector: 1) the displacement of a singlet or a multiplet, defined as a shift displacement $(\Delta \delta)$; and 2) the enantiomeric splitting of a singlet or a multiplet, defined as a shift non-equivalence $\left(\Delta \delta^{*}\right)$. At least one type of shift could be observed for each resonance, and sometimes both occurred in combination.

In order to evaluate the potential of these chiral selectors, the ${ }^{1} \mathrm{H}-\mathrm{NMR}$ spectra of $0.014 \mathrm{M}$ IBP solutions containing different concentrations of selectors were studied. The amounts of selectors added to the racemic IBP solutions were as follows:

- $\beta$-CD: $0.0018,0.0053,0.0088,0.013$ and $0.015 \mathrm{M}$.

- MD17: 0.0008, 0.0042, 0.0067, 0.013 and $0.018 \mathrm{M}$.

- MD19: 0.0015, 0.0074, 0.0101, 0.0129, 0.0150, 0.0185, 0.0215 and $0.0352 \mathrm{M}$.

The expansion of the ${ }^{1} \mathrm{H}-\mathrm{NMR}$ spectra of IBP upon the addition of different concentrations of the chiral selectors, shown in Figure 4, revealed that the $\mathrm{F}$ proton signal demonstrated shift displacement coupled with shift nonequivalence. A clear splitting of the doublet was observed in these spectra as a result of the complexation.

The reversible exchange between the complexed and the free solute was rapid on the NMR time scale, and the NMR proton signals were the average values of the free and complexed solutes [16]. This demonstrated that differences in the complexation-induced shifts in the NMR spectra resulted from the enantiomeric composition of the solute. Therefore, the signal splitting of the IBP revealed the chiral recognition capability of the hosts. In addition, the structures of the complexes formed by the enantiomeric guest and the hosts induced clear nonequivalent complexation-induced shifts and provided chiral discrimination.

Many NMR studies of chiral selectors have shown that the protons closest to the binding site have greater enantiomeric differences in their complexation-induced shifts $[17,18]$. It should also be noted in the present study that in the complexes of IBP with both MDs, the doublet signal of the IBP F protons was the only one split, whereas in the complex with $\beta$-CD the shielding shift was accompanied by the splitting of the doublet signals of the A and $\mathrm{F}$ protons. Therefore, the $\mathrm{F}$ protons (methyl protons attached to the asymmetric carbon atom of IBP) are likely
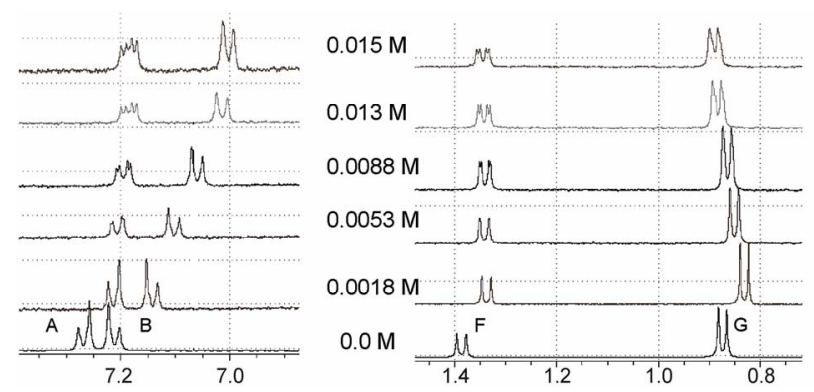

(a)
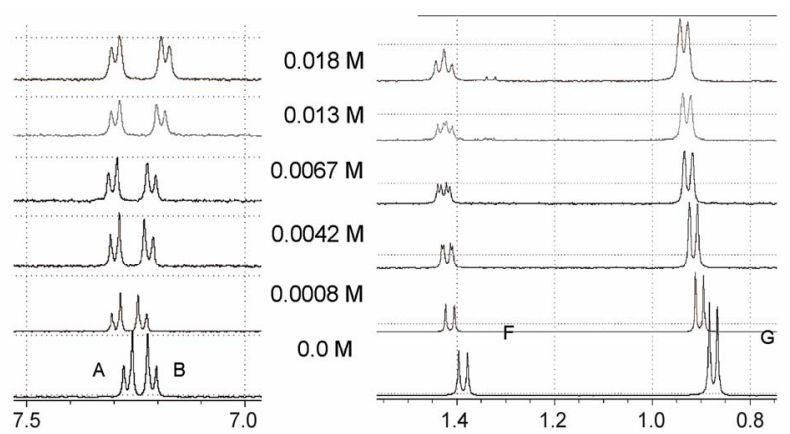

(b)
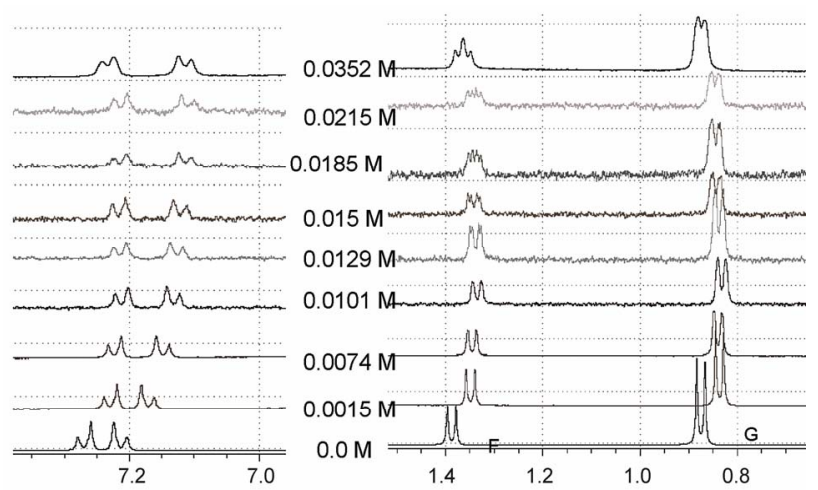

(c)

Figure 4. Expansions of IBP ${ }^{1} \mathrm{H}-\mathrm{NMR}$ spectra upon the addition of different concentrations of (a) $\beta$-CD; (b) MD17; (c) MD19.

to be in close proximity to the host when the complexes are formed. Similar results have been reported by Oh et al. [19], who suggested that in the presence of hydroxypropyl- $\beta$-cyclodextrin, the multiplicity of the methyl proton signal of IBP might arise from the differences in the shielding extent of the diastereoisomeric pairs.

The resolution of enantiomers can usually be enhanced by optimization of the selector concentration. For all cases examined, the magnitude of the splitting obtained was strongly affected by the molar ratio IBP/selector (Figure 4) and showed differences in shifts related to the concentration of the different selectors. Generally, increasing the concentration of the host reagent promoted the formation of diastereomeric complexes and enhanced the enantiomeric discrimination visible in the NMR spec- 
tra, with the concentrations necessary to separate the IBP enantiomers being $0.0053 \mathrm{M}, 0.0042 \mathrm{M}$ and $0.0074 \mathrm{M}$ for $\beta$-CD, MD17 and MD19, respectively. However, in these cases, the resolution of the IBP enantiomeric forms was not enhanced with increasing concentration of the selectors, because higher concentrations caused broadening and superposition of the resonances.

Differences in the magnitude of the complexation-induced shifts, in part, reflected the chiral recognition capability of the host, with $\beta$-CD showing the highest shifts in most of the signals of IBP. Also, the shifts due to MD19 were greater compared to those of MD17. These results are depicted in Tables 3-5.

The results of these studies can be applied in an analytical approach to develop and optimize methods for the chromatographic separation, detection and determination of the enantiomeric composition of IBP based on the diasteroisomeric formation assay. Thus, reliable correlations between NMR experiments and chromatographic enantioseparations would be expected.

\subsubsection{Evidence of Interaction between IBP and MDs}

The 2D-ROESY experiments were used to obtain useful information concerning the supramolecular system structures present in solution. The bidimensional spectra of IBP-MD17 and IBP-MD19 systems allowed us to establish clear intermolecular dipolar interactions between protons at distances of less than $5 \AA$. The MD protons were assigned according to the report of Wangsakan et al. [20].

The observed intermolecular proximities revealed the formation of complexes between IBP and each ligand, but suggested that the molecular interaction inside the MD helix might be different for each MD. An expansion of the ROESY spectrum of IBP-MD17, shown in Figure 5(a), revealed several intermolecular cross-peaks between the protons 2, 5 and 6 of MD and protons $\mathrm{A}, \mathrm{B}$ and $\mathrm{G}$ of IBP. The 2D-ROESY spectrum of IBP-MD19 is shown in Figure 5(b). Strong cross-peaks were observed between proton $\mathrm{G}$ of IBP and the protons 2, 5 and 6 of MD, while very weak dipolar correlations were seen between

Table 3. Complexation induced chemical shifts $(\Delta \delta)$ of racemic IBP in the presence of different concentrations of $\beta$-CD.

\begin{tabular}{cccccc}
\hline \multirow{2}{*}{ Proton of IBP } & \multicolumn{5}{c}{$\beta$-CD concentration } \\
\cline { 2 - 5 } & $0.0018 \mathrm{M}$ & $0.0053 \mathrm{M}$ & $0.088 \mathrm{M}$ & $0.013 \mathrm{M}$ & $0.015 \mathrm{M}$ \\
\hline A & -0.0351 & $-0.0460^{*}$ & $-0.0531^{*}$ & $-0.0642^{*}$ & $-0.0665^{*}$ \\
B & -0.0501 & -0.0947 & -0.1329 & -0.1798 & -0.1933 \\
D & -0.0214 & -0.0214 & -0.0176 & -0.0160 & -0.0162 \\
E & -0.0301 & -0.0275 & -0.0206 & -0.0151 & -0.0148 \\
F & -0.0289 & -0.0297 & $-0.0242^{*}$ & $-0.0261^{*}$ & $-0.0261^{*}$ \\
G & -0.0225 & -0.0079 & 0.0103 & 0.0295 & 0.0333 \\
\hline
\end{tabular}

$\Delta \delta=\delta_{\text {complex }}-\delta_{\text {free, }}$, shift non-equivalence was observed coupled with shift displacement.

Table 4. Complexation induced chemical shifts $(\Delta \delta)$ of racemic IBP in the presence of different concentrations of MD17.

\begin{tabular}{cccccc}
\hline \multirow{2}{*}{ Proton of IBP } & \multicolumn{5}{c}{ MD17 concentration } \\
\cline { 2 - 5 } & $0.0008 \mathrm{M}$ & $0.0042 \mathrm{M}$ & $0.0067 \mathrm{M}$ & $0.0131 \mathrm{M}$ & $0.0179 \mathrm{M}$ \\
\hline A & 0.0271 & 0.0300 & 0.0346 & 0.0294 & 0.0290 \\
B & 0.0225 & 0.0082 & 0.0015 & -0.0197 & -0.0292 \\
D & 0.0154 & 0.0177 & 0.0217 & 0.0157 & 0.0225 \\
E & 0.0273 & 0.0303 & 0.0353 & 0.0341 & 0.0380 \\
F & 0.0273 & $0.0332^{*}$ & $0.0399^{*}$ & $0.0378^{*}$ & $0.0399^{*}$ \\
G & 0.0288 & 0.0412 & 0.0517 & 0.0553 & 0.0610 \\
\hline
\end{tabular}

$\Delta \delta=\delta_{\text {complex }}-\delta_{\text {free }}{ }^{*}$ shift non-equivalence was observed coupled with shift displacement.

Table 5. Complexation induced chemical shifts $(\Delta \delta)$ of racemic IBP in the presence of different concentrations of MD19.

\begin{tabular}{|c|c|c|c|c|c|c|c|c|}
\hline \multirow{2}{*}{ Proton of IBP } & \multicolumn{8}{|c|}{ MD19 concentration } \\
\hline & $0.0015 \mathrm{M}$ & $0.0074 \mathrm{M}$ & $0.0101 \mathrm{M}$ & $0.0129 \mathrm{M}$ & $0.0150 \mathrm{M}$ & $0.0185 \mathrm{M}$ & $0.0215 \mathrm{M}$ & $0.0352 \mathrm{M}$ \\
\hline A & -0.0359 & -0.0384 & -0.0465 & -0.0448 & -0.0453 & -0.0466 & -0.0464 & -0.0494 \\
\hline B & -0.0378 & -0.0568 & -0.0707 & -0.0765 & -0.0835 & -0.0904 & -0.0945 & -0.1124 \\
\hline $\mathrm{D}$ & -0.0280 & -0.0312 & -0.0395 & -0.0384 & -0.0388 & -0.0459 & -0.0413 & -0.0387 \\
\hline $\mathrm{E}$ & -0.0378 & -0.0409 & -0.0451 & -0.0437 & - & -0.0493 & -0.0561 & -0.0511 \\
\hline G & -0.0343 & -0.0269 & -0.0323 & -0.0277 & -0.0251 & -0.0215 & -0.0209 & -0.0141 \\
\hline
\end{tabular}

$\Delta \delta=\delta_{\text {complex }}-\delta_{\text {free }},{ }^{*}$ shift non-equivalence was observed coupled with shift displacement. 

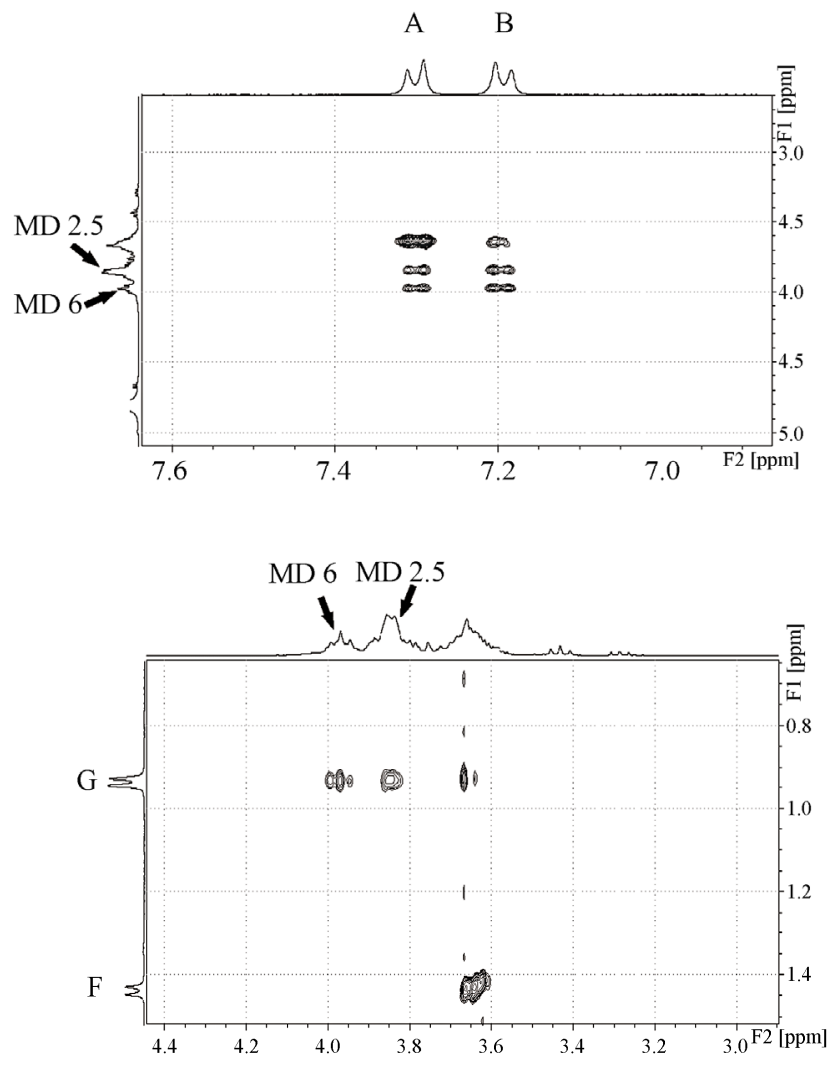

(a)
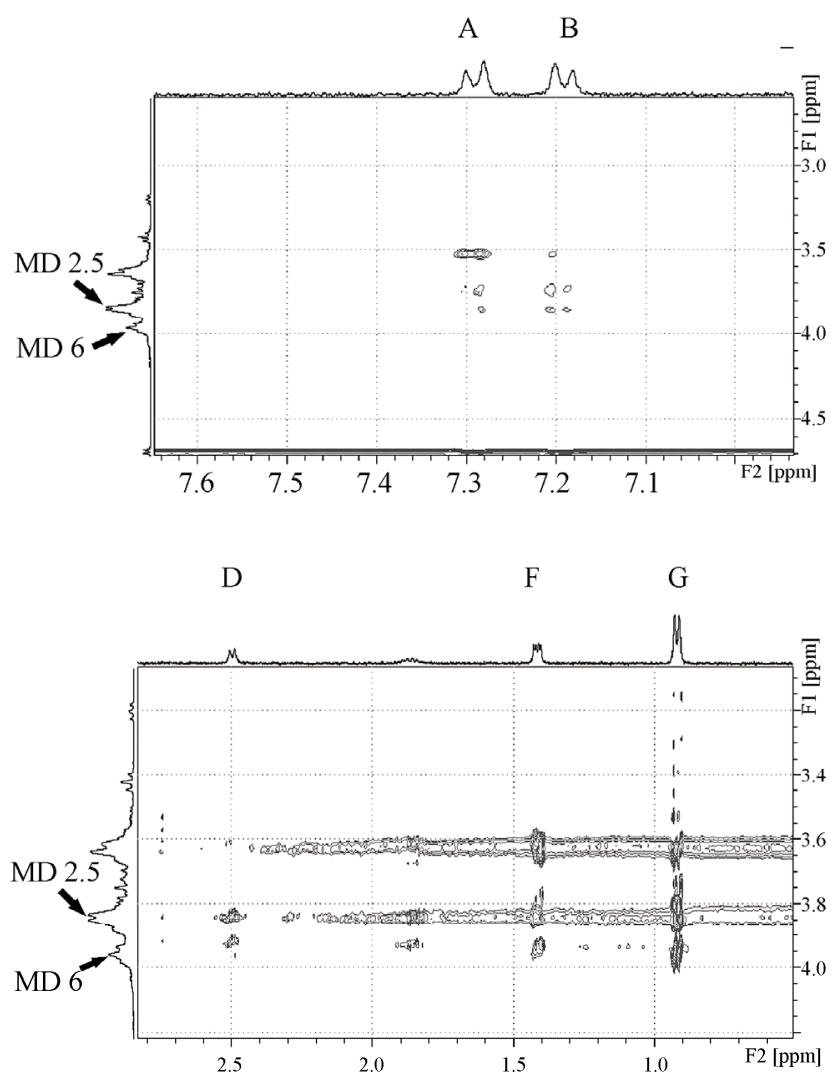

(b)

Figure 5. Representative regions of 2D-ROESY spectrum showing the intermolecular proximities between IBP and (a) MD17 and (b) MD19.

these host protons and the aromatic $\mathrm{A}$ and $\mathrm{B}$ protons of IBP. On the other hand, the D proton of IBP showed a preference for the protons 2,5 and a weak cross-peak with the proton 6 of MD, whereas the F proton of IBP displayed intermolecular cross-peaks with the proton 6 of $\mathrm{MD}$ and very weak dipolar correlations with the protons 2, 5 of MD.

Interestingly, MD19 caused intermolecular cross-peaks in more signals of IBP compared to MD17. However, the most intense cross-peaks were those between the MD17 protons and the protons of IBP. In conclusion, our data may be considered to be direct evidence of the different hydrophobic interactions of IBP with MD17 and MD19.

\subsubsection{Determination of the Apparent Stability Constants}

The $\mathrm{K}_{\mathrm{c}}$ values were determined from the NMR titration experiments (Scott's method) by taking into account the most marked shift variations. Therefore, the signal assigned to the aromatic A and B protons for the system IBP- $\beta$-CD were used, as well as the signal of the proton $\mathrm{G}$ for the IBP-MD17 system and the signals of the protons B and G for the IBP-MD19 system.

Linear Scott plots were obtained for all the complexes studied ( $r>0.97)$. Choosing different guest hydrogen atoms among those discriminated by the chiral selector gave different $\mathrm{K}_{\mathrm{c}}$ values, with the average $\mathrm{K}_{\mathrm{c}}$ being 206 $\mathrm{M}^{-1}, 579 \mathrm{M}^{-1}$ and $168 \mathrm{M}^{-1}$ for the IBP- $\beta$-CD, IBP-MD17, and IBP-MD19 systems, respectively.

The differences found between the determined $\mathrm{K}_{\mathrm{c}}$ values obtained by solubility and NMR studies arose from the use of distinct methods of determination, due to the fact that different techniques measure different physico-chemical parameters. However, both experiments revealed that the interaction IBP-MD occurred more efficiently with MD17.

It is thought that the IBP hydrophobic phenyl group leaves the hostile hydrophilic environment of the aqueous media to the hydrophobic CD cavity or MD helix. In addition, the penetration of the phenyl group into the hydrophobic cavity or helix is considered to be a thermodynamically driven hydrophobic interaction process. From a thermodynamic point of view, Rekharsky and Innoue [21] argue that stronger binding between the guest and the host results in a loss of chiral recognition. In our case, IBP was more strongly bound with MD17 than with $\beta$-CD or MD19, which may have affected the chiral recognition capability of the host. 


\subsection{Characterization of the Complexes}

\subsubsection{Differential Scanning Calorimetry (DSC) and Thermogravimetric Analysis (TGA)}

The DSC and TGA profiles of IBP, MDs, physical mixtures and freeze-dried systems are shown in Figures 6(A) and (B), respectively. IBP exhibited a sharp endothermic melting peak at $78.11^{\circ} \mathrm{C}$. Also, the weight loss that initiated over $100^{\circ} \mathrm{C}$, shown by the TGA curve, could not be exclusively due to thermal decomposition, revealing the tendency of the drug to undergo sublimation. The trace of MD17 showed a broad endotherm in the range of $35^{\circ} \mathrm{C}-165^{\circ} \mathrm{C}$ with a maximum at around $91^{\circ} \mathrm{C}$, corresponding to a dehydration process, as was determined by the weight loss of $5.2 \%$ registered by TGA. The MD19 curve exhibited a very broad endothermic effect, between $40^{\circ} \mathrm{C}$ and $185^{\circ} \mathrm{C}$, which reached a maximum at around $104^{\circ} \mathrm{C}$ due to a dehydration process and was associated with a mass fraction loss of $5.6 \%$.

The DSC curve of the IBP-MD17 physical mixture displayed a melting peak at $77.56^{\circ} \mathrm{C}$, which reflected the presence of remaining drug crystals unincorporated in the carrier. In contrast, the complete disappearance of the endothermic peak of free IBP was observed for the same system obtained by the freeze-drying method, which might have been due to a complexation of the drug. In addition, the freeze-dried and physical mixture system TGA curves showed that the dehydration stage contained only $6.9 \%$ and $6.8 \%$ of water, respectively, compared to $5.1 \%$ of MD17. This behavior indicates that most of the water molecules in the MD17 helix cavity were replaced by IBP during the complexation process.

The DSC curves of the IBP-MD19 systems obtained by the freeze-dried technique and physical mixture dem- onstrated the complete disappearance of the IBP peak, indicating molecular encapsulation of the drug. Interestingly, the IBP-MD17 and IBP-MD19 freeze-dried systems and physical mixtures TGA curves showed enhancement of IBP thermal stability in the solid state.

\subsubsection{Fourier Transform-Infrared Spectroscopy (FT-IR)}

Generally, carboxylic acids are characterized in FT-IR spectroscopy by bands from the $\mathrm{OH}$ stretch, the $\mathrm{OH}$ inand out-of-plane stretching, and the $\mathrm{C}=\mathrm{O}$ asymmetrical stretching bands. All these bands are sensitive to the hydrogen bonding of the $\mathrm{COOH}$ group that exists either as the unbounded monomer or as the carboxylic acid dimer. The carboxylic acid monomer has a characteristic strong absorbance between 1800 and $1740 \mathrm{~cm}^{-1}$, but when two carbonyl groups are presented with a symmetry center as in the case of a carboxylic acid dimer, this band shifts to $1720-1680 \mathrm{~cm}^{-1}$. Also, when the carbonyl is hydrogen bonded, but not dimerised as in alcohol-carbonyl bonding, a band is seen near $1730-1705 \mathrm{~cm}^{-1}[22,23]$.

Segments of the FT-IR spectra of IBP, MDs, physical mixtures and freeze-dried systems are shown in Figure 7. The FT-IR spectrum of IBP showed all the characteristic bands of the drug, including a stretching band at 1720.21 $\mathrm{cm}^{-1}$ corresponding to the carbonyl group, which confirms that it exists in the dimeric form. For the IBPMD17 freeze-dried system, the IBP $\mathrm{C}=\mathrm{O}$ band was shifted to $1727.96 \mathrm{~cm}^{-1}$ with a new band appearing at $1747.05 \mathrm{~cm}^{-1}$, suggesting the formation of hydrogen bonds between IBP and MD17 by means of intermolecular hydrogen bond breakdown of the IBP crystals. Taking into account these results, we propose that the hydrogen bonding state of IBP changed from the dimeric form to a

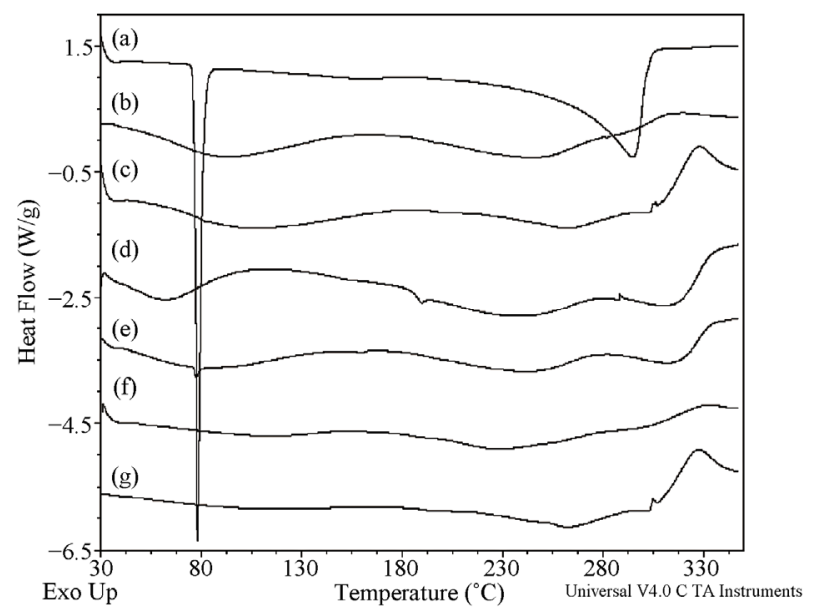

(A)

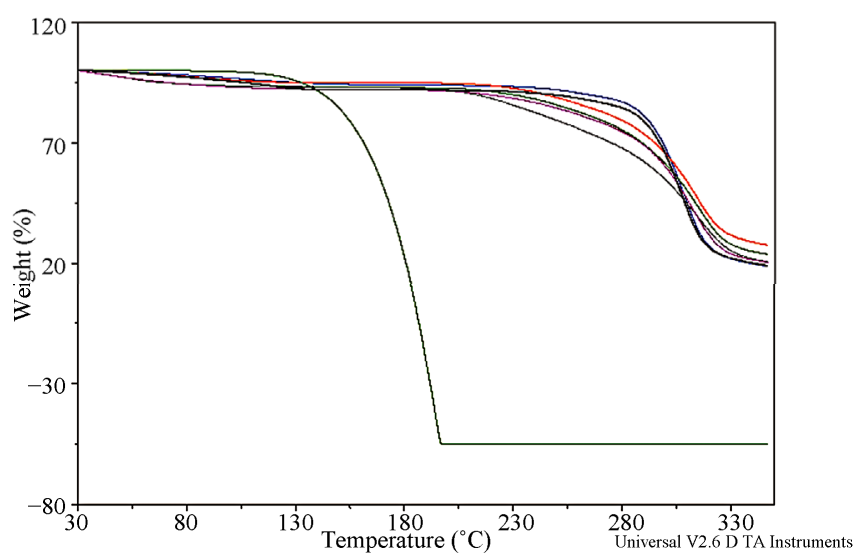

(B)

Figure 6. (A) DSC curves of: (a) IBP; (b) MD17; (c) MD19; (d) IBP-MD17 freeze-dried; (e) IBP-MD17 physical mixture; (f) IBP-MD19 freeze-dried; (g) IBP-MD19 physical mixture; (B) TGA curves of IBP (green), MD17 (red), MD19 (blue), IBP-MD17 freeze-dried (gray), IBP-MD17 physical mixture (lime), IBP-MD19 freeze-dried (fuchsia), IBP-MD19 physical mixture (black). 


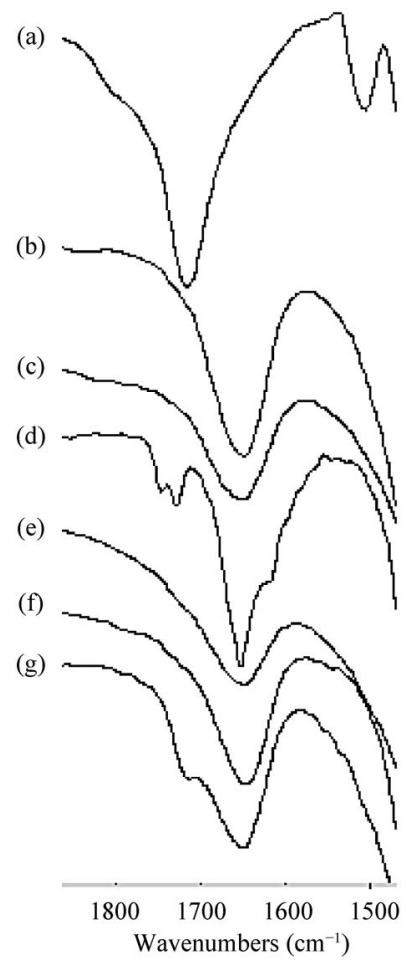

Figure 7. FT-IR spectra of: (a) IBP; (b) MD17; (c) MD19; (d) IBP-MD17 freeze-dried (e) IBP-MD17 physical mixture, (f) IBP-MD19 freeze-dried; (g) IBP-MD19 physical mixture. carbonyl-hydroxyl $(\mathrm{C}=\mathrm{O}-\mathrm{HO})$ hydrogen bonded form in the presence of MD17, and that the ratio of the absorbance of the $1747.05 \mathrm{~cm}^{-1}$ and $1727.96 \mathrm{~cm}^{-1}$ bands provided a measure of the relative amounts of both the hydroxyl and dimeric forms. Similar interactions have also been previously observed with hydroxypropyl methylcellulose [24], polyoxyethylene alkyl ethers [25] and polyethylene glycol [26].

In the case of the IBP-MD17 physical mixture and the IBP-MD19 freeze-dried system spectra, the characteristic $\mathrm{C}=\mathrm{O}$ band disappeared. On the other hand, in the spectrum of the IBP-MD19 physical mixture, this band shifted to the lower frequency of $1714.24 \mathrm{~cm}^{-1}$, which could be indicating the existence of intermolecular hydrogen bonding between IBP and MD19 leading to restrictions within the MD helix. Bearing in mind these spectral changes, there is undoubtedly clear evidence of interactions between IBP and the hosts MD17 and MD19, suggesting the formation of complexes in the solid state.

\subsubsection{Scanning Electron Microscopy (SEM)}

Morphological changes were investigated by SEM (Figure 8). IBP appeared as needle-shaped crystals, MD17 consisted of hollow spherical entities with large particles containing smaller particles, and MD19 appeared as agglomerated irregularly shaped particles.
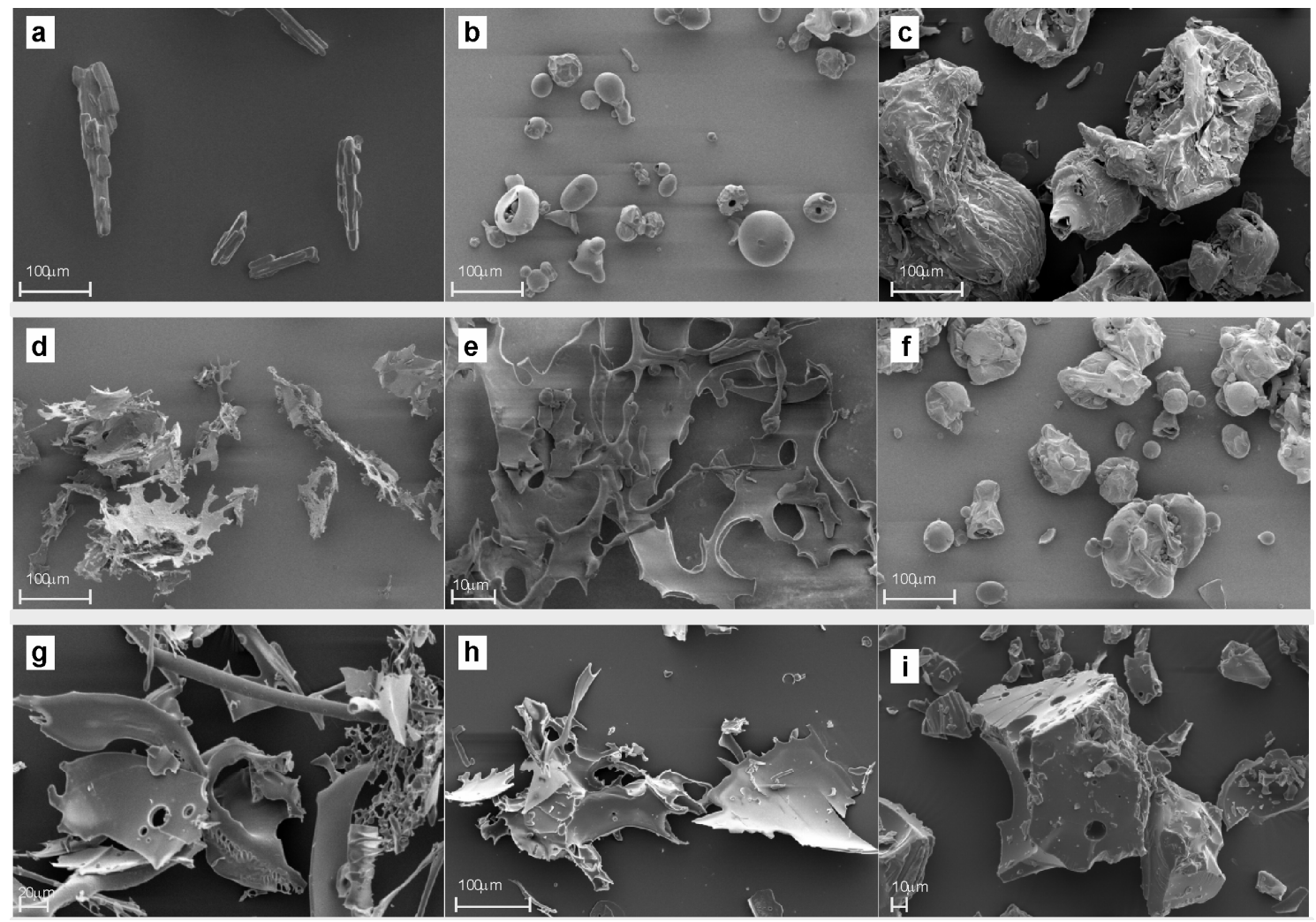

Figure 8. Scanning electron microphotographs of: (a) IBP; (b) MD17; (c) MD19; (d), (e) IBP-MD17 freeze-dried; (f) IBP-MD17 physical mixture; (g), (h) IBP-MD19 freeze-dried; (i) IBP-MD19 physical mixture. 
In the SEM images of the IBP-MD17 and IBP-MD19 freeze-dried systems, the original morphology of the raw materials disappeared and it was impossible to differentiate the single components. However, by magnification of the images, it was possible to observe in detail that these amorphous structures had both smooth and rough surfaces. These drastic changes in particle shape and aspect in the freeze-dried complexes can be taken as a proof of the formation of new solid phases, as previously noted in the DSC analysis.

The IBP-MD17 and IBP-MD19 physical mixtures consisted of amorphous entities, with some similarities with the original morphology of MDs and containing some particles adhered to their surfaces. Thus, an apparent interaction of both components of the system occurred.

\subsubsection{X-Ray Diffractometry (XRD)}

Powder XRD is a useful method for detecting complexation in powder or microcrystalline states. The X-ray diffraction patterns of the raw materials, the freeze-dried binary systems and the physical mixtures are shown in Figure 9.

The diffraction pattern of IBP exhibited high intensity reflections with a series of characteristic peaks, which are indicative of its crystalline character. For MDs, a hollow pattern was recorded, revealing their amorphous state. In the case of physical mixtures, the XRD patterns had few reflections in the angle range of $5^{\circ}-40^{\circ}(2 \theta)$, indicating that the drug was no longer present in a crystalline form and suggesting a possible interaction. On the other hand, the XRD patterns of the freeze-dried binary systems were completely diffuse, with an absence of intense sharp peaks. The disappearance of the IBP diffraction pattern may have been due to the formation of true complexes of an amorphous nature in which the IBP were trapped in

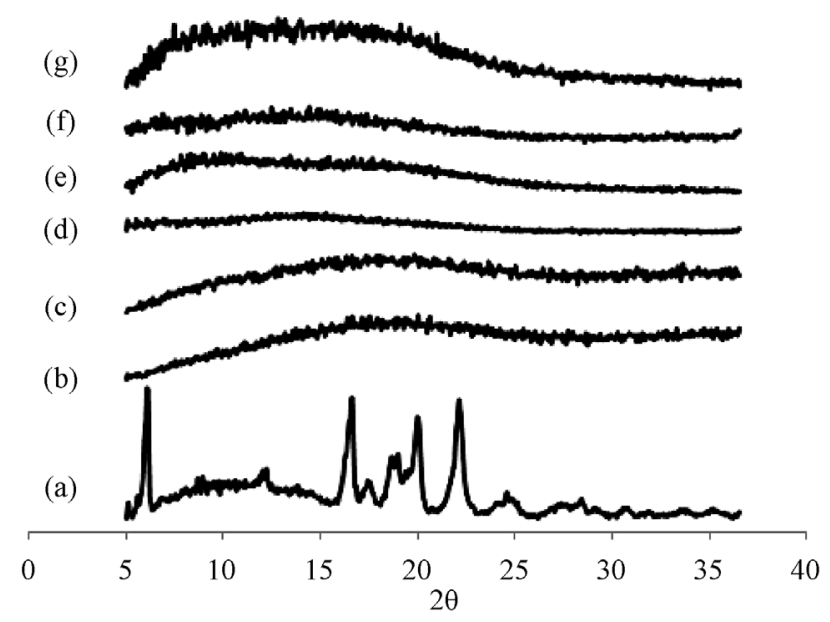

Figure 9. X-Ray powder diffraction patterns of: (a) IBP; (b) MD17; (c) MD19; (d) IBP-MD17 freeze-dried; (e) IBPMD17 physical mixture; (f) IBP-MD19 freeze-dried; (g) IBP-MD19 physical mixture. the MDs. Thus, the complexation of IBP and MDs produced new solid phases that had less crystalline structures compared to that of the pure form of IBP.

\section{Conclusions}

In this study, we have characterized the interactions of the ligands MD17 and MD19 with the guest IBP, resulting in the formation of different binary complexes. These distinct modes of complexation were confirmed by means of phase solubility analysis and ${ }^{1} \mathrm{H}-\mathrm{NMR}$ and 2D-ROESY experiments in solution, as well as by DSC, TGA, FT-IR, SEM and XRD in the solid state.

The solubility of IBP was improved by the addition of MDs and making $\mathrm{pH}$ adjustments to the complexation medium. In particular, MD17 provided the greatest solubilization effect in the $\mathrm{pH} 2.0$ solution. Furthermore, the NMR results showed that the interaction mode of IBP inside the MD helix might be different with each MD. However, in the presence of both MDs, the drug showed chiral discrimination, indicating that these linear ligands act as chiral selectors and that the diastereomeric complexes obtained are suitable to be used for chiral recognition.

Finally, this study may be regarded as an important step towards optimizing IBP assays related with enantiomeric analytical aspects, and should have an impact on the chiral recognition of MDs that might be extended to separation techniques.

\section{Acknowledgements}

Financial support from Fondo para la Investigación Científica y Tecnológica (FONCYT) Préstamo BID 1728/OC-AR PICT 1376, the Consejo Nacional de Investigaciones Científicas y Técnicas (CONICET) and the Secretaría de Ciencia y Técnica de la Universidad Nacional de Córdoba (SECyT-UNC) is greatly acknowledged. The assistance from Dr Gloria M. Bonetto in performing NMR measurements and her helpful discussion of the spectra are highly appreciated. We also thank Ferromet S.A. (agent of Roquette in Argentina) for their donation of Glucidex ${ }^{\circledR}$ IT 19 DE19. We would like to thank Dr. Paul Hobson, native speaker, for revision of the manuscript.

\section{REFERENCES}

[1] J. D. Higgins, T. P. Gilmor, S. A. Martellucci, R. D. Bruce and H. G. Brittain, "Ibuprofen," Analytical Profiles of Drug Substances, Vol. 27, Academic Press, London, 2001.

[2] S. S. Adams, P. Bresloff and C. G. Manson, "Pharmacological Differences between the Optical Isomers of Ibuprofen: Evidence for Metabolic Inversion of the (-)-Isomer," Journal of Pharmacy and Pharmacology, Vol. 28, 
No. 3, 1976, pp. 256-257. doi:10.1111/j.2042-7158.1976.tb04144.x

[3] C. J. Nuñez Aguero, C. M. Escobar Llanos, D. Diaz, C. Jaime and R. Garduño Juarez, "Chiral Discrimination of Ibuprofen Isomers in -Cyclodextrin Inclusion Complexes: Experimental (NMR) and Theoretical (MD, MM/GBSA) Studies," Tetrahedron, Vol. 62, No. 17, 2006, pp. 41624172. doi:10.1016/j.tet.2006.02.010

[4] P. Valderrama and R. J. Poppi, "Second Order Standard Addition Method and Fluorescence Spectroscopy in the Quantification of Ibuprofen Enantiomers in Biological Fluids," Chemometrics and Intelligent Laboratory Systems, Vol. 106, No. 2, 2011, pp. 160-165.

doi:10.1016/j.chemolab.2010.05.012

[5] K. Busch, I. Swamidoss, S. Fakayode and M. Busch, "Determination of the Enantiomeric Composition of Some Molecules of Pharmaceutical Interest by Chemometric Analysis of the UV Spectra of Cyclodextrin GuestHost Complexes," Analytica Chimica Acta, Vol. 525, No. 1, 2004, pp. 53-62. doi:10.1016/j.aca.2004.07.066

[6] K. Ozoemena, R. Stefan, J. van Staden and H. AboulEnein, "Utilization of Maltodextrin Based Enanti-Oselective, Potentiometric Membrane Electrodes for the Enantioselective Assay of S-Perindopril," Talanta, Vol. 62, No. 4, 2004, pp. 681-685.

doi:10.1016/j.talanta.2003.08.035

[7] M. Shamsipur, L. Dastjerdi, S. Haghgoo, D. Armspach, D. Matt and H. Aboul-Enein, "Chiral Selectors for Enantioresolution and Quantitation of the Antidepressant Drug Fluoxetine in Pharmaceutical Formulations by 19F NMR Spectroscopic Method," Analytica Chimica Acta, Vol. 601, No. 1, 2007, pp. 130-138. doi:10.1016/j.aca.2007.08.017

[8] J. Wang and L. Wang, "Structures and Properties of Commercial Maltodextrins from Corn, Potato, and Rice Starches," Starch/Starke, Vol. 52, 2000, pp. 296-304. doi:10.1002/1521-379X(20009)52:8/9<296::AID-STAR2 96>3.0.CO;2-A

[9] L. Dokic-Baucal, P. Dokic and J. Jakovljevic, "Influence of Different Maltodextrins on Properties of O/W Emulsions," Food Hydrocolloids, Vol. 18, No. 2, 2004, pp. 233-239. doi:10.1016/S0268-005X(03)00068-7

[10] H. Soini, M. Stefansson, M. Riekkola and M. Novotny, "Maltooligosaccharides as Chiral Selectors for the Separation of Pharmaceuticals by Capillary Electrophoresi," Analytical Chemistry, Vol. 66, 1994, pp. 3477-3484. doi:10.1021/ac00092a028

[11] T. Higuchi and K. A. Connors, "Phase-Solubility Techniques," In: C. N. Reilly, Ed., Advances in Analytical Chemistry and Instrumentation, Wiley-Interscience, New York, Vol. 4, 1965, pp. 117-212.

[12] H. Benesi and J. Hildebrand, "A Spectrophotometric Investigation of the Interaction of Iodine with Aromatic Hydrocarbons," Journal of the American Chemical Society, Vol. 71, No. 8, 1949, pp. 2703-2707. doi:10.1021/ja01176a030

[13] R. L. Scott, "Some Comments on the Benesi-Hildebrand Equation," Recueil des Travaux Chimiques, Vol. 75, No. 7, 1956, pp. 787-789. doi:10.1002/recl.19560750711
[14] K. H. Fromming and J. Szejtli, "Cyclodextrin Inclusion Complexes," Cyclodextrins in Pharmacy, Kluwer Academic Publishers, Dodrecht/Boston/London, 1994.

[15] G. Granero, C. Garnero and M. Longhi, "The Effect of $\mathrm{pH}$ and Triethanolamine on Sulfisoxazole Complexation with Hydroxypropyl- $\beta$-Cyclodextrin," European Journal of Pharmaceutical Sciences, Vol. 20, No. 3, 2003, pp. 285-293. doi:10.1016/S0928-0987(03)00202-1

[16] G. Endresz, B. Chankvetadze, D. Bergenthal and G. Blaschke, "Comparative Capillary Electrophoretic and Nuclear Magnetic Resonance Studies of the Chiral Recognition of Racemic Metomidate with Cyclodextrin Hosts," Journal of Chromatography A, Vol. 732, No. 1, 1996, pp. 133-142. doi:10.1016/0021-9673(95)01244-3

[17] K. Kano, H. Hasegawa and M. Miyamura, "Chiral Recognition of Dipeptide Methyl Esters by an Anionic $\beta$ Cyclodextrin," Chirality, Vol. 13, 2001, pp. 474-482. doi:10.1002/chir.1064

[18] C. F. Dignam, L. A. Randall, R. D. Blacken, P. R. Cunningham, S. G. Lester, M. J. Brown, S. C. French, S. E. Aniagyei and T. J. Wenzel, "Carboxymethylated Cyclodextrin Derivatives as Chiral NMR Discriminating Agents," Tetrahedron: Asymmetry, Vol. 17, No. 8, 2006, pp. 1199-1208. doi:10.1016/j.tetasy.2006.04.006

[19] I. Oh, M. Y. Lee, Y. B. Lee, S. C. Shin and I. Park, "Spectroscopic Characterization of Ibuprofen/2-Hydroxypropyl- $\beta$-Cyclodextrin Inclusion Complex," International Journal of Pharmaceutics, Vol. 175, No. 2, 1998, pp. 215-223. doi:10.1016/S0378-5173(98)00286-5

[20] A. Wangsakan, D. McClements, P. Chinachoti and C. Dickinson, "Two-Dimensional Rotating-Frame Overhauser Spectroscopy (ROESY) and ${ }^{13} \mathrm{C}$ NMR Study of the Interactions between Maltodextrin and an Anionic Surfactant," Carbohydrate Research, Vol. 339, No. 6, 2004, pp. 1105-1111. doi:10.1016/j.carres.2004.01.019

[21] M. V. Rekharsky and Y. Innoue, "Complexation and Chiral Recognition Thermodynamics of 6-Amino-6-deoxy-beta-cyclodextrin with Anionic, Cationic, and Neutral Chiral Guests: Counterbalance between van der Waals and Coulombic Interactions," Journal of the American Chemical Society, Vol. 124, 2002, pp. 813-826. doi:10.1021/ja010889z

[22] N. Najib, M. Suleiman and A. Malakh, "Characteristics of the in Vitro Release of Ibuprofen from Polyvinylpyrrolidone Solid Dispersions," International Journal of Pharmaceutics, Vol. 32, No. 2-3,1986, pp. 229-236. doi:10.1016/0378-5173(86)90183-3

[23] N. Colthup, L. Daly and S. Wiberly, "Introduction to Infrared and Raman Spectroscopy," Academic Press, New York, 1990, pp. 163-181. doi:10.1016/S0378-5173(00)00603-7

[24] M. Iervolino, B. Capello, S. Raghavan and J. Hadgraft, "Penetration Enhancement of Ibuprofen from Supersaturated Solutions through Human Skin," International Journal of Pharmaceutics, Vol. 212, No. 1, 2001, pp. 131141.

[25] E. Park, S. Chang, M. Hahn and S. Chi, "Enhancing Effect of Polyoxyethylene Alkyl Ethers on the Skin Permeation of Ibuprofen," International Journal of Pharma- 
ceutics, Vol. 209, No. 1, 2000, pp. 109-119.

doi:10.1016/S0378-5173(00)00559-7

[26] T. Shakhtshneider, M. Vasilchenko, A. Politov and V. Boldyrev, "The Mechanochemical Preparation of Solid
Disperse Systems of Ibuprofen-Polyethylene Glycol," International Journal of Pharmaceutics, Vol. 130, No. 1, 1996, pp. 25-32. 